DOI: $10.24850 / j-$ tyca-2020-03-02

Artículos

\title{
Modelación no estacionaria de la magnitud y frecuencia de las crecidas en el Alto Cauca mediante índices climáticos y de operación de embalse Non-stationary modeling of the magnitude and frequency of floods in Alto Cauca through climatic and reservoir operation indexes
}

Karime Sedano1, ORCID: https://orcid.org/0000-0002-6336-5387

Yesid Carvajal-Escobar², ORCID: orcid.org/0000-0002-2014-4226

Jesús López-De la Cruz3, ORCID: https://orcid.org/0000-0001-82306414

Félix Francés ${ }^{4}$, ORCID: https://orcid.org/0000-0003-1173-4969

${ }^{1}$ Universidad del Valle, Cali, Valle del Cauca, Colombia, karimesedano@gmail.com

2Universidad del Valle, Cali, Valle del Cauca, Colombia yesid.carvajal@correounivalle.edu.co

universidad de Colima, Facultad de Ingeniería Civil, Colima, México, jlopez71@ucol.mx

4Universitat Politècnica de València, Valencia, España, ffrances@upv.es 


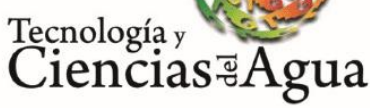

2020, Instituto Mexicano de Tecnología del Agua

Open Access bajo la licencia CC BY-NC-SA 4.0

(https://creativecommons.org/licenses/by-nc-sa/4.0/)

Autora para correspondencia: Karime Sedano, karimesedano@gmail.com

\section{Resumen}

A través de los modelos aditivos generalizados de localización, escala y forma se estudian los cambios en la frecuencia y magnitud de las crecidas anuales en el río Cauca, localizado en el suroccidente de Colombia. El análisis de frecuencias de crecidas no estacionario incorpora dos índices macroclimáticos y un índice antrópico, que permite asumir los cambios en el almacenamiento de agua en el embalse y el porcentaje de área tributaria regulada, como factores que inciden en la capacidad de la represa para laminar las crecidas. Los resultados muestran que forzamientos que dependen del Índice Multivariado del Fenómeno El Niño Oscilación del Sur (ENSO, por sus siglas en inglés) y del índice de embalse propuesto, cuando son aplicados a los parámetros de la función de distribución, mejoran la calidad de ajuste y la descripción de la variabilidad temporal de la serie de tiempo de crecidas. Frente al análisis convencional, los modelos no estacionarios indican diferencias significativas en los caudales asociados con cierto periodo de retorno y en el riesgo de fallo de un caudal de diseño en función de la vida útil. La principal conclusión es que desde 1986, en la estación de aforo Juanchito, los caudales exhiben un incremento en magnitud que está asociada inequívocamente con eventos de la fase fría del fenómeno ENSO. Por lo tanto, los modelos no estacionarios proveen información de interés para el embalse, su estrategia de regulación de caudales altos y en la gestión del riesgo de inundaciones. 
Palabras clave: análisis de frecuencias de crecidas, no estacionariedad, fenómeno ENSO, Colombia, alteración antrópica.

\section{Abstract}

In this study, changes in the frequency and magnitude of annual floods in the Cauca River (Southwest Colombia) are modeled using a nonstationary framework by means of the Generalized Additive Models of Localization, Scale and Shape. Non-stationary flood frequency analysis incorporates two climatic indices and an anthropic index that allows us to assume that changes in the water reservoir and the percentage of regulated tributary area are factors which disturb the capacity of the dam to withhold the flood. The results highlight the role of the El Niño Southern Oscillation (ENSO) phenomenon and the proposed Reservoir Index, as significant covariates in the parameters of the selected distributions. The dependence of model parameters on covariates improves the model's capacity for representing temporal variability of the flood regime. Nonstationary models indicate significant differences in the flow associated with a specific return period, and in the failure risk of flow design, depending on the working life, in contrast to classical stationary models. The main conclusion is that since 1986 in the gauging station Juanchito, flooding has shown a gradual increase in magnitude, which is unambiguously associated with the cold phase of the ENSO phenomenon. Therefore, the non-stationary models provide valuable information on the reservoir, its regulation strategy for high flow, and for flood risk management in the Cauca river basin. 
Keywords: Floods frequency analysis, non-stationary, El Niño South Oscillation Phenomenon, Colombia, anthropogenic interference.

Recibido: 26/09/2018

Aceptado: $23 / 08 / 2019$

\section{Introducción}

La variabilidad climática asociada con El Niño Oscilación del Sur (ENSO, por sus siglas en inglés) tiene un profundo impacto ambiental y socioeconómico en Colombia. La fase fría del ENSO genera emergencias por inundaciones y deslizamientos de tierra que pueden afectar a más de 500000 personas, con una frecuencia de 2 a 4 años. En el periodo $1950-$ 2018 se reportan 177 desastres naturales en el país; 45\% de los casos corresponde a inundaciones (EM-DAT, 2018). Además, con relación a Latinoamérica, Colombia presenta la mayor tasa de emergencias recurrentes provocadas por fenómenos naturales (más de 600 reportes por año); esto se debe no sólo a las condiciones ambientales naturales sino a que $84.7 \%$ de la población está localizada en áreas expuestas a dos o más peligros naturales (Banco Mundial, 2014). 
Ciencias $₫$ Agua
2020, Instituto Mexicano de Tecnología del Agua

Open Access bajo la licencia CC BY-NC-SA 4.0

(https://creativecommons.org/licenses/by-nc-sa/4.0/)

El Análisis de Frecuencias de Crecidas (AFC) es el modelo estadístico más utilizado para estimar el caudal de una crecida y/o su frecuencia de ocurrencia, con el fin de dimensionar estructuras hidráulicas y gestionar los recursos hídricos en una cuenca. El análisis hace inferencias acerca de una variable hidrológica, adoptando la hipótesis que las observaciones son independientes e idénticamente distribuidas (hipótesis i.i.d.), por lo tanto no hay cambios sistemáticos en la media o varianza que determinen la aparición de tendencias durante el periodo de las observaciones ni en las extrapolaciones que sobre dichos datos se realizan. No obstante, hay evidencias sobre los efectos de los cambios ambientales globales y de variabilidad climática en cuanto a la alteración del comportamiento de variables hidrológicas en el espacio y tiempo; motivo por el cual desde hace más de una década se desarrollan metodologías estadísticas que abordan el AFC no estacionario.

Estudios antecedentes sobre los efectos de la variabilidad climática en el régimen hidrológico de Colombia destacan: 1) fuertes correlaciones entre ENSO y las variables hidrológicas, por ejemplo, mayor variabilidad en los caudales mensuales de los ríos Cauca y Magdalena; 2) tendencias de incremento/disminución en el tiempo de la temperatura del aire, precipitación y caudales mensuales, especialmente en la región Andina, y 3) la función de distribución de probabilidades de la serie de caudales máximos es afectada por ambas fases de ENSO (Ávila, Guerrero, Escobar, \& Justino, 2019; Carvajal, Jiménez, \& Materon, 1998; Gutiérrez \& Dracup, 2001; Jiménez-Cisneros et al., 2014; Poveda, 2004; Poveda \& Álvarez, 2012; Poveda et al., 2002; Poveda, Waylen, \& Pulwarty, 2006; Puertas \& Carvajal, 2008). Debido a las anteriores evidencias, el análisis del riesgo hidrológico asociado con el fenómeno ENSO es clave para la gestión de 
Tecnología y

Ciencias $\stackrel{\Xi}{\unlhd}$ gua
2020, Instituto Mexicano de Tecnología del Agua

Open Access bajo la licencia CC BY-NC-SA 4.0

(https://creativecommons.org/licenses/by-nc-sa/4.0/)

inundaciones en Colombia, en particular en la región Andina, pues en ella se concentra $80 \%$ de la población.

En la actualidad sigue vigente el debate de adoptar o no el supuesto de estacionariedad requerido en la estadística hidrológica (Matalas, 1997; Milly et al., 2015; Milly et al., 2008; Montanari \& Koutsoyiannis, 2014). Los discursos son antagónicos. Por un lado, se hace un llamado a incorporar la no estacionariedad en la modelación hidrológica para representar mejor la realidad, mientras otros sostienen que las múltiples fuentes de incertidumbre a considerar hacen que la aproximación estocástica sea aún robusta. Aunque no existe un consenso sobre la mejor metodología de análisis, hay puntos en común, como la necesidad de incrementar la comprensión del sistema clima-agua-sociedad, disminuir las fuentes de incertidumbre de los modelos, y sobre todo desarrollar metodologías de análisis más robustas para proponer soluciones adaptativas eficaces frente a las tendencias de cambio del riesgo hidrológico.

Muchas investigaciones abordan la no estacionariedad en las variables hidrológicas, logrando desarrollar metodologías no estacionarias para el análisis de frecuencias de eventos extremos, y proponer adaptaciones a los conceptos periodo de retorno y riesgo en el diseño hidrológico (Khaliq, Ouarda, Ondo, Gachon, \& Bobée, 2006; Salas \& Obeyskera, 2014). Los modelos no estacionarios más estudiados incorporan el forzamiento de tendencias usando covariables externas, como índices de variabilidad climática; datos de precipitación y temperatura; señales de cambio global a escala reducida; tasas de urbanización, índices de embalse, etcétera. Este tipo de análisis pretende establecer vínculos entre procesos físicos del entorno y los cambios en la 
Tecnología y

Ciencias $₫$ Agua
2020, Instituto Mexicano de Tecnología del Agua

Open Access bajo la licencia CC BY-NC-SA 4.0

(https://creativecommons.org/licenses/by-nc-sa/4.0/)

distribución de probabilidades asociada con los registros anuales de caudales máximos. Hay abundantes referencias sobre el uso de los modelos aditivos generalizados en los parámetros de localización, escala y forma (GAMLSS, por sus siglas en inglés), como una alternativa flexible tanto en la selección de la función de distribución como en el tipo de tendencias sobre sus parámetros (Ahn \& Palmer, 2016; Córdoba, Palomino, Gámiz, Castro, \& Esteban, 2015; Vasiliades, Galiatsatou, \& Loukas, 2015; Villarini \& Strong, 2014). Otros trabajos en este sentido emplean modelos bayesianos y el análisis espectral singular (EscalanteSandoval, \& Garcia-Espinoza, 2014; Lima \& Lall, 2011; Poveda \& Álvarez, 2012).

Todo lo anterior motiva la modelación estadística no estacionaria de las crecidas en Colombia. Se elige evaluar los caudales máximos anuales del río Cauca, en la cuenca del Valle del Alto Cauca, ubicado en la región Andina. La zona presenta un régimen bimodal, con precipitaciones entre 1300 y 3000 mm, y su llanura aluvial tiene 840 km² susceptibles a inundaciones periódicas, por lo que desde 1985 los caudales son regulados por un embalse. El río Cauca es de interés, pues una de las regiones más urbanizadas de Colombia; además, entre 1950 y 2015, se han registrado 14 eventos históricos de inundaciones del río Cauca (Enciso, Carvajal, \& Sandoval, 2016); seis de los cuales ocurrieron después de la construcción del embalse (en 1988, 1997, 1999, 2008, 2010 y 2011) y la misma cantidad de eventos coinciden con el fenómeno La Niña. Los datos analizados corresponden a dos estaciones hidrométricas de interés, empleando el modelo GAMLSS propuesto por Rigby y Stasinopoulos (2005). 
La hipótesis yace en considerar si el uso de modelos no estacionarios para el caso de estudio presenta diferencias significativas frente al análisis estacionario y tiene por objetivo demostrar que incorporar forzamientos climáticos (a través de varios índices macroclimáticos asociados con el trópico suramericano) y de la actividad humana (usando un índice específico de operación de embalse) resultan adecuados como términos aditivos que describen los cambios en la frecuencia y magnitud de los caudales máximos anuales. Los modelos estadísticos evaluados pueden ser de interés para evidenciar oportunidades de gestión de los recursos hídricos, en especial las reglas de operación de un embalse frente a eventos de crecidas, en beneficio de la ciudad de Cali (tercera ciudad más poblada de Colombia). A continuación, se presenta una caracterización del área de estudio, así como la metodología empleada en el análisis de frecuencias no estacionario. Después se analizan los resultados obtenidos y se resumen algunas conclusiones.

\section{Área de estudio}

La cuenca Valle Alto Cauca se ubica en el suroccidente colombiano. El río Cauca sigue una dirección sur-norte en un valle interandino (Figura 1). En los primeros $153 \mathrm{~km}$ de recorrido, el cauce desciende $2.8 \mathrm{~km}$ hasta llegar al embalse Salvajina, cuya área de drenaje es $3652 \mathrm{~km}^{2}$. En el 
Tecnología y

Ciencias $\stackrel{\unlhd}{\unlhd}$ Agua
2020, Instituto Mexicano de Tecnología del Agua

Open Access bajo la licencia CC BY-NC-SA 4.0

(https://creativecommons.org/licenses/by-nc-sa/4.0/)

tramo posterior, la cuenca alcanza $5111 \mathrm{~km}^{2}$ en la estación de aforo La Balsa y finalmente el área de interés cierra en la estación hidrométrica Juanchito, cubriendo un total de $8556 \mathrm{~km}^{2}$. Ambas estaciones son seleccionadas teniendo en cuenta: 1) la proximidad a la salida del embalse; 2) la estación Juanchito es el punto de control de la operación de volúmenes en la represa Salvajina, y 3) Juanchito se localiza en las afueras de la ciudad de Cali, que es la tercera ciudad más poblada de Colombia, con 2.4 millones de habitantes.
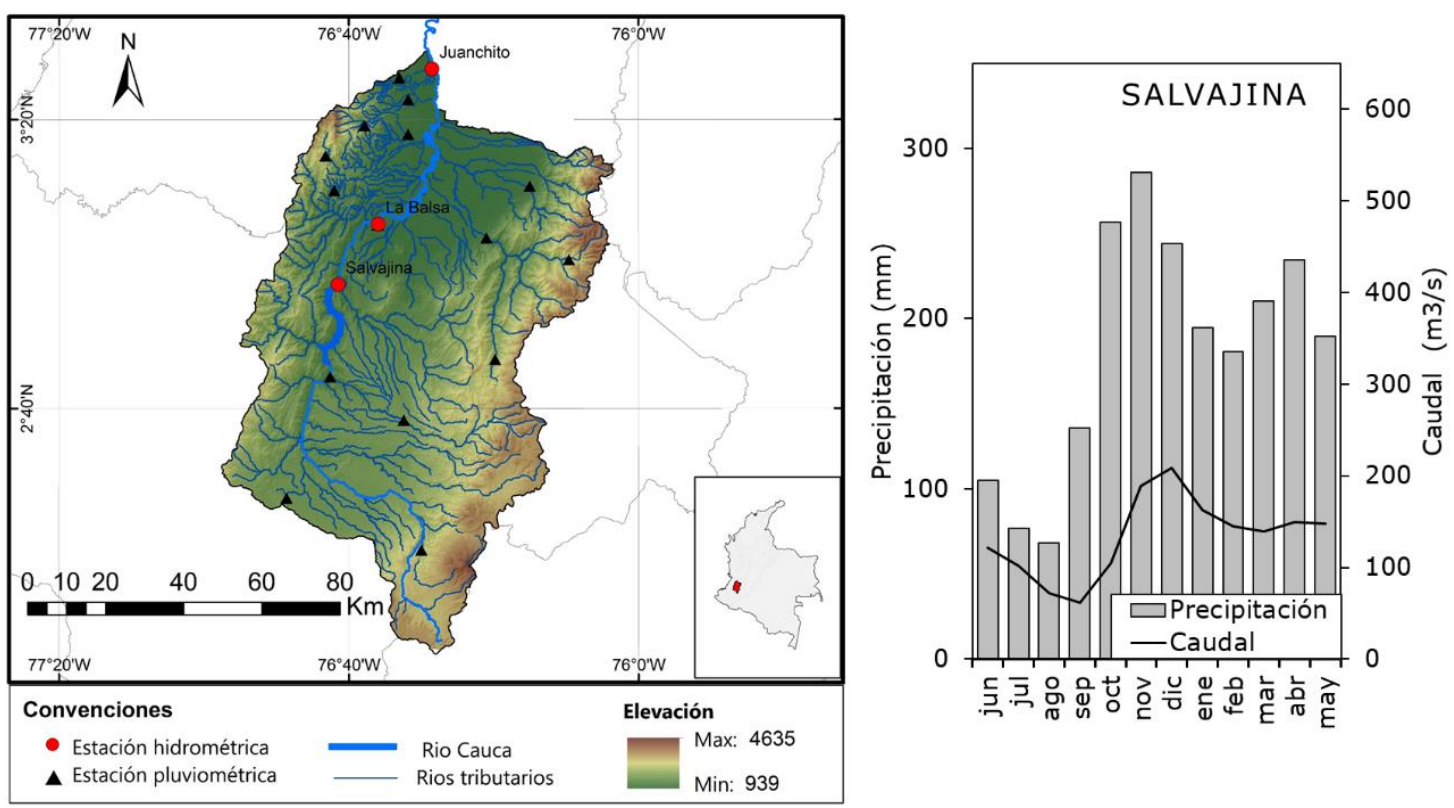

Figura 1. Localización de la cuenca Valle Alto Cauca y la red hidrológica (izquierda). Precipitación mensual en el área tributaria al embalse y el caudal afluente a Salvajina (derecha y arriba). Almacenamiento mensual en el embalse Salvajina y caudal promedio en Juanchito, estación objetivo de regulación (derecha y abajo). 
El embalse Salvajina posee una capacidad máxima de almacenamiento de $848 \mathrm{hm}^{3}$; sus objetivos de operación son el control de inundaciones y estiajes, garantizando caudales entre 900 y 130 m³/s; 95\% del tiempo en la estación Juanchito (Sandoval, Ramirez, \& Santacruz, 2011).

El régimen hidrológico en la región es fuertemente afectado por la variabilidad climática vinculada con el doble paso de la Zona de Convergencia Intertropical (ZCIT); la orografía; los procesos que ocurren en los océanos Atlántico, mar Caribe, el Pacífico y la Amazonia; pero sobre todo a la influencia del fenómeno ENSO en sus fases extremas (García, Botero, Bernal, Ardila, \& Piñeros, 2012; Puertas \& Carvajal, 2008; Rueda \& Poveda, 2006).

La precipitación media anual en la cuenca alta del río Cauca es 1 $900 \mathrm{~mm}$, y predomina un ciclo bimodal con mayores lluvias en los periodos marzo-abril-mayo (MAM) y septiembre-octubre-noviembre (SON) (Figura 1, derecha) (Sandoval \& Ramirez, 2007). Se han reportado fuertes conexiones entre los caudales medios mensuales de diversos ríos del occidente colombiano con el Índice Oceánico El Niño-ONI, y otras señales de cambio en la temperatura superficial del océano Pacífico; la Oscilación Decadal del Pacífico-PDO, con señales climáticas compuestas por variables atmosféricas y oceánicas, como el Índice Multivariado ENSO-MEI; y por variables exclusivamente atmosféricas, como la Corriente en Chorro del Chocó-CCC, y el Índice Oscilación del Sur (SOI, por sus siglas en inglés), entre otros (Jiménez-Cisneros et al., 2014; Poveda, Jaramillo, \& Vallejo, 2014; Poveda et al., 2006). 
2020, Instituto Mexicano de Tecnología del Agua

\section{Materiales}

Todas las variables hidrológicas y climáticas se construyen considerando que el año hidrológico en Colombia inicia el $1^{\circ}$ de junio del año $i$ y finaliza el 31 de mayo del año $i+1$. Se define como periodo común de registro 1965-2015, pues los datos faltantes son inferiores al 5\%. Por último, es necesario resaltar que los datos del año 1985 se adoptan como atípicos debido al proceso de llenado del embalse, y se excluyen de las series hidrológicas y climáticas. La información fue suministrada por la Corporación Autónoma Regional del Valle del Cauca, en adelante CVC. La serie temporal analizada es el caudal diario máximo registrado por año

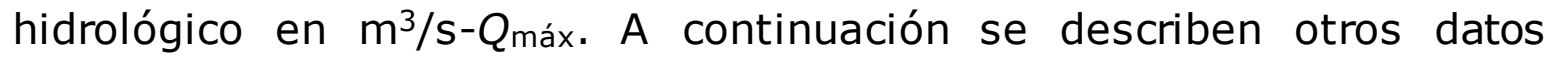
empleados en este trabajo.

\section{Índice de embalse}

López y Francés (2013) proponen un índice de embalse IE (Ecuación (1)), que establece el grado de alteración del régimen hidrológico en función 
Tecnología y

Ciencias $\stackrel{\Xi}{\unlhd}$ gua
2020, Instituto Mexicano de Tecnología del Agua

Open Access bajola licencia CC BY-NC-SA 4.0

(https://creativecommons.org/licenses/by-nc-sa/4.0/)

del porcentaje de área no regulada y el porcentaje de escurrimiento medio que no puede ser retenido en el embalse:

$$
\operatorname{IE}(t)=\sum_{i=1}^{N} \frac{A_{R_{i}}(t)}{A_{s}} \cdot \frac{C_{R i}(t)}{C_{s}}, \text { para } i=1,2,3, \ldots, N .
$$

Donde $A_{R_{i}}(t)$ es el área tributaria al embalse en $\mathrm{km}^{2}$, en el año $t$; $A_{S}$, el área tributaria a la estación en $\mathrm{km}^{2} ; C_{S}$, el volumen promedio escurrido en la cuenca hasta la estación $S$ en el año $t$ y en $\mathrm{hm}^{3} ; C_{R}$, la capacidad total de almacenamiento del embalse en $\mathrm{hm}^{3}$ en el año $t$, y $\mathrm{N}$ es el número de embalses aguas arriba de la estación. Para una cuenca con un único embalse en cabecera y sin otros cambios antrópicos significativos en el tiempo (p. ej., trasvase, aumento/disminución de embalses, etc.) que afecten las variables $A_{R_{i}}(t) A_{R_{i}}(t)$ y $C_{R i}(t)$, este índice funciona como una variable discreta o señal de cambio que es intensa/débil en función de la proximidad de la estación de aforo de interés a la represa y ha sido empleado en la modelación estadística no estacionaria de crecidas (Liang, Jing, Wang, Binquan, \& Zhao, 2017; Machado et al., 2015).

Los impactos de un embalse en la laminación de las crecidas dependen de la capacidad disponible en el embalse, el tamaño de la obra en relación con el aporte hídrico de la cuenca, los usos del agua almacenada y de los niveles de agua en el embalse antes de la avenida, etcétera (Moreno, Begueria, Garcés, \& García, 2003). Por capacidad disponible se entiende el volumen libre o diferencia entre la capacidad máxima de almacenamiento y el volumen de agua en un momento dado. 
Los cambios en la capacidad disponible pueden limitar/favorecer la gestión del caudal máximo anual en la estación objetivo. Por lo tanto, en este trabajo se propone ajustar la Ecuación (1), remplazando la relación entre la capacidad de reservorio y el escurrimiento medio, por el coeficiente de volumen de reserva anual $D_{(t)}=\left(C_{R(t)}-V_{a(t)}\right) / C_{R(t)}$ que determina la proporción de volumen disponible frente a la capacidad del reservorio $\overline{V_{R}}(t)$, de tal forma que el índice $I E_{2}$ es:

$$
I E_{2}(t)=\sum_{i=1}^{n} \frac{A_{R_{i}}(t)}{A_{s}} \cdot \frac{C_{R(t)}-V_{a}(t)}{C_{R}(t)}, \text { para } i=1,2, \ldots, N
$$

Donde $V_{a(t)}$ es el volumen promedio anual almacenado en el embalse Salvajina, estimado con datos de batimetrías realizadas por la CVC en 1985, 2003 y 2011, y el registro de niveles en el embalse al final del día. Con el cambio, además del efecto de disminución en la capacidad de regulación a medida que el área tributaria a la estación de aforo aumenta (establecido por la relación de áreas), el índice considera la gestión de volúmenes como condición que influye en los caudales máximos anuales observados.

\section{Índices de variabilidad climática}


Teniendo en cuenta que las fases de ENSO son definidas por el Organismo Nacional Estadounidense del Océano y la Atmósfera (NOAA), en función del Índice Oceánico El Niño (ONI), en este trabajo se adopta el valor promedio anual de ONI como criterio para clasificar cada año hidrológico de la siguiente forma: un año es considerado La Niña, si el valor promedio anual de ONI $\leq-0.30$; años El Niño ocurre cuando ONI $\geq-0.30$; un año es definido como normal siempre que $-0.30<$ ONI $<0.30$. Los índices climáticos de baja frecuencia empleados en la modelación corresponden a los valores promedio anuales del Índice Oceánico El Niño-ONI o media móvil trimestral de las anomalías de la temperatura superficial del Pacífico en la región Niño3-4 y el Índice Multivariado ENSO-MEI, que corresponde a la primera componente principal de un conjunto de señales oceánicas y atmosféricas en toda la región del Pacífico tropical. Todos los valores son estimados a partir de información publicada en NOAA (2017).

\section{Modelos de aditivos generalizados en los parámetros de localización, escala y forma-GAMLSS}

Los modelos GAMLSS adoptan que la variable respuesta $Y$ ( $\left.Q_{\text {máx }}\right)$ posee una función de distribución de probabilidad acumulativa, cuyos parámetros pueden ser función de una o más variables explicativas (índices climáticos y de embalse). Es decir, para yt variables independientes en el tiempo $t=1,2, \ldots, n$ existe una función $F_{Y}\left(y_{t} \mid \theta_{t}\right)$, 
donde los parámetros $\theta_{t}=\left(\mu_{t}, \sigma_{t}, v_{t}, \tau_{t}\right)$ pueden cambiar en función de un conjunto de $m$ variables explicativas $X_{m t}=\left[x_{1 t}, x_{2 t}, \ldots, x_{m t}\right]$, a través de una función de enlace monotónica $g_{k}(\cdot)$ presentada en la Ecuación (3) (Stasinopoulos, Rigby, Vlasios, Heler, \& Bastiani De, 2015):

$$
\mathrm{g}_{\mathrm{k}}\left(\theta_{\mathrm{k}}\right)=\eta_{k}=\mathrm{X}_{\mathrm{k}} \beta_{\mathrm{k}}+\sum_{\mathrm{j}=1}^{\mathrm{m}_{\mathrm{k}}} \mathrm{h}_{\mathrm{jk}}\left(\mathrm{x}_{\mathrm{jk}}\right)
$$

Donde $\theta_{k}$ y $\eta_{k}$ son vectores de longitud $n ; X_{k}$ es una matriz de covariables de orden $n \times m ; \beta_{k}$ es un vector de parámetros de longitud $m$; $h_{j k}\left(x_{j k}\right)$ representan el suavizado en los parámetros de la distribución, y $x_{j k}$ es un vector de covariables para $j=1,2, \ldots, m$.

Como función de suavizado se evalúan los B-splines penalizados, $p b()$ que son partes polinómicas usadas en modelos aditivos, con la ventaja que minimizan los grados de libertad $(d f)$ del modelo, a través de diferentes métodos, como máxima verosimilitud (ML), GAIC, etcétera. Se puede consultar más información en Stasinopoulos et al. (2015).

\section{Periodo de retorno no estacionario}

El periodo de retorno $T$ es un indicador de la rareza de una crecida; es decir, el tiempo medio que transcurre hasta que, por primera vez, una 
crecida excede un valor dado $\left(y_{\mathrm{p} 0}\right)$. Es usado comúnmente para definir el caudal de diseño de una obra hidráulica o para referenciar el nivel de amenaza de inundación. Teniendo en cuenta que la estadística de dicho suceso sigue una distribución geométrica, la probabilidad de que la primera crecida exceda a $y_{q 0}$ en el tiempo $x$ es $f(x)=p_{x} \prod_{t=1}^{x-1}\left(1-p_{t}\right), x=$ $1,2, \ldots, x_{\max }$ (Salas \& Obeyskera, 2014), donde $f(1)=p_{1}$ y $x_{\text {max }}$ es el tiempo en el que $p_{t}$ se convierte en 1 . Si la probabilidad $p_{t}$ es constante en el tiempo $f(x)=P(X=x)=(1-p)^{x-1} p$, el periodo de retorno o tiempo de espera previsto (EWT) es:

$$
T=E(X)=1 / p=1 /(1-\mathrm{q})
$$

Donde $\mathrm{p}$ y $q$ son la probabilidad de excedencia y no excedencia, respectivamente. Cuando la probabilidad $p_{t}$ es no estacionaria, entonces el periodo de retorno cambia en el tiempo:

$$
T_{t}=\frac{1}{p_{t}}=\frac{1}{\left[1-F_{Y}^{-1}\left(y_{\mathrm{p} 0}, \theta_{t}\right)\right]}
$$

Los valores de la probabilidad de excedencia $p_{t}$ son obtenidos de $p_{t}=1-F_{Y}^{-1}\left(y_{\mathrm{p} 0}, \theta_{t}\right)$ usando la información disponible; $y_{\mathrm{p} 0}$ es un caudal de referencia y el vector de parámetros $\theta_{t}$ varía en función de un conjunto de covariables $X_{m t}$ (Obeyskera \& Salas, 2016). Teniendo en cuenta la Ecuación (5) en el diseño hidráulico y en la gestión de los recursos hídricos surgen cuestiones como ¿Cuál es el valor de $T$ de un evento histórico, 
asociado con un modelo no estacionario de crecidas? ¿Cuál es el caudal de diseño si el valor de $T$ es variable en el tiempo? Debido a esto, Salas y Obeyskera (2014) proponen determinar el valor $T$ no estacionario, usando una distribución geométrica no homogénea:

$$
T_{E W T}=E(X)=1+\sum_{x=1}^{x_{\max }} \prod_{t=1}^{x}\left(1-p_{t}\right), x=1,2, \ldots, x_{\max }
$$

Donde los valores $p_{t}=1-q_{t}$ se obtienen de $p_{t}=1-F_{Y}\left(y_{q 0}, \theta_{t}\right)$, para el $y_{q 0}$ preestablecido y el modelo estadístico no estacionario. Salas Obeysekera y Vogel (2018), así como Serinaldi (2015) presentan otra forma simplificada de estimar el periodo de retorno no estacionario, basado en el concepto de análisis económico "riesgo anual medio" (AAR, por sus siglas en inglés) expresado como: $\operatorname{AAR}(n)=\bar{p}=(1 / n)\left(p_{1}+p_{2}+\cdots+\right.$ $\left.p_{n}\right)$, donde se adopta que para un periodo $n$, el riesgo puede ser descrito por la media de la secuencia de probabilidades de excedencia $\left(p_{1}, p_{2}, \ldots, p_{n}\right)$; debido a esto, $T$ no estacionario equivale a:

$$
T_{A A R}=\bar{T}=1 / \operatorname{AAR}(n)=1 / \bar{p}
$$

Serinaldi (2015) sostiene que uno de los indicadores más importantes en la planeación y el diseño de obras hidráulicas es el riesgo de fallo $R$ o probabilidad $R$ de que al menos una vez se observe un evento extremo $y_{q 0}$ durante la vida útil $n$ de una obra, siendo esta expresión la más adecuada o directa para definir el caudal de diseño frente a un nivel de riesgo aceptable, ya que depende de la probabilidad de excedencia y 
no del periodo de retorno, cuando existen condiciones i.i.d. $R=P(X \leq n)=$ $F_{X}(n)=\sum_{x=1}^{n} f(x)=1-(1-p)^{n} \quad$ (Chow, Maidment, \& Mays, 1994); sin embargo, en condiciones de no estacionariedad, Salas et al. (2018) usan los conceptos tiempo de espera previsto y riesgo anual medio para determinar el riesgo de fallo no estacionario:

$$
\begin{gathered}
R_{E W T}(n)=1-\prod_{t=1}^{n}\left(1-p_{t}\right), \\
\overline{R_{A A R}}=1-(1-\bar{p})^{n}
\end{gathered}
$$

Donde $p_{t}$ y $\bar{p}$ es la probabilidad de excedencia y la probabilidad de excedencia media multianual, respectivamente. $R_{E W T}$ y $R_{A A R}$ se resuelven de manera numérica.

\section{Metodología}

La modelación estadística de la frecuencia y magnitud de los extremos hidrológicos se basa en los modelos a ditivos generalizados de localización, escala y forma GAMLSS. Todos los cálculos pueden ejecutarse en la plataforma $R$, usando los paquetes GAMLSS gratuitos disponibles. El análisis tiene las etapas de la Figura 2. 


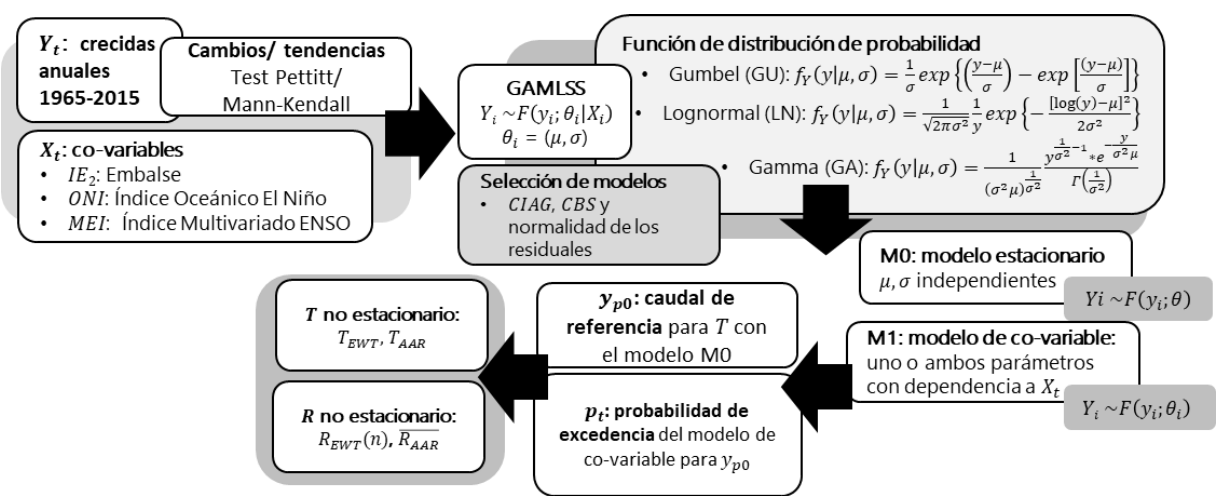

Figura 2. Diagrama de flujo de las etapas que componen el análisis de frecuencias no estacionario.

\section{Identificación de cambios y tendencias en el régimen de crecidas}

Las pruebas estadísticas no paramétricas de Mann-Kendall (Mann, 1945; Yue \& Wang, 2002) y Pettitt (1979) son aplicadas a las series temporales para analizar la estacionariedad. Además, con el objetivo de abordar los efectos de la operación del embalse, las series de crecidas máximas anuales se dividieron en los periodos 1965-1984 y 1986-2015, representando así el régimen no regulado y el régimen alterado, respectivamente. 


\section{Ajuste y selección de modelos estadísticos no estacionarios}

Los modelos GAMLSS se emplean para forzar cambios graduales en el parámetro de localización $(\mu)$ en función de las covariables ONI, MEI e IE2. Las funciones de distribución de probabilidad seleccionadas son Gumbel (GU), Lognormal (LN) y Gamma (GA). Todas poseen dos parámetros $(\mu, \sigma)$, son adecuadas para contrarrestar los efectos de la asimetría positiva, común en series de extremos hidrológicos. Los resultados se centrarán en los mejores modelos obtenidos y no en el conjunto de modelos evaluados.

Se analizan dos tipos de modelos estadísticos: los modelos estacionarios (M0), donde ambos parámetros de la distribución son independientes; y los modelos de covariables externas (M1), en los que el parámetro de localización $\mu$ puede depender linealmente de uno o varios índices climáticos, del índice de embalse o de la combinación de ambos tipos de variables explicativas. La selección de los modelos se basa en el Criterio de Información de Akaike Generalizado (CIAG) y el Criterio Bayesiano de Schwartz (CBS) (Ecuación (10) y Ecuación (11)):

$$
C I A G=-2 \hat{\imath}+2(k \cdot d f)
$$




$$
C B S=-2 \hat{\imath}+\ln (n) *(k \cdot d f)
$$

Donde $\hat{l}=\ln (M L)$; la máxima verosimilitud del modelo es $M L$, luego $k$ : penalidad requerida asociada con el número de parámetros de la distribución y $d f$ : grados de libertad. En este trabajo se adopta una penalidad $k=3.0$, de tal forma que el grado de complejidad del modelo no degrade la capacidad de describir el comportamiento de la serie y conserve en lo posible el principio de parsimonia.

En ausencia de un estadístico para determinar la bondad de ajuste de los modelos GAMLSS, Stasinopoulos et al. (2015) proponen verificar la normalidad de los residuales; para ello se evalúa el coeficiente de correlación de Filliben y el comportamiento de los gráficos sin tendencia de los residuales (worm-plot). Esto asegura que el modelo seleccionado represente la parte sistemática y que la información remanente (residuales) sea ruido blanco.

\section{El periodo de retorno en modelos no estacionarios}

Para comparar los resultados obtenidos en los modelos M0 y M1 se emplean las formulaciones EWT y AAR. El cálculo inicia definiendo un caudal $y_{\mathrm{p} 0}$ con el modelo estacionario; luego se emplea el modelo no estacionario M1 para estimarla probabilidad de excedencia; se aplican las 
ecuaciones (4) a (9) para determinar el riesgo hidrológico en términos de $T$ y $R$ no estacionario, considerando, entre otros aspectos: el momento en el que $p_{t}$ se convierte en uno; la longitud $n$ de registros, y el patrón de cambio predominante en M1. Es importante mencionar que para cada estación, $R$ es estimado para el caudal de diseño de $T=100$ años y diferentes valores $n$ de vida útil.

\section{Resultados y discusión}

\section{Alteración del régimen de crecidas}

A continuación se analizan los cambios en el régimen de crecidas en La Balsa y Juanchito.

La Tabla 1 confirma que los caudales en La Balsa disminuyen durante el periodo de registros y dicha tendencia es significativa para $\boldsymbol{\alpha}=$ 0. 05. Además, existen puntos de cambio en la media en 1975 y 1984, que coinciden con cambios de fase de la PDO y puesta en marcha del embalse. Debido a la proximidad de la estación a la salida del reservorio es posible asociar la no estacionariedad con la alteración antrópica. La Figura 3b muestra el decrecimiento en la magnitud de las crecidas de Juanchito 
Tecnología y

Ciencias $\stackrel{\unlhd}{\unlhd}$ Agua
2020, Instituto Mexicano de Tecnología del Agua

Open Access bajo la licencia CC BY-NC-SA 4.0

(https://creativecommons.org/licenses/by-nc-sa/4.0/)

entre 1965 y 1984, pero dicho cambio carece de significancia estadística (Tabla 1). Por el contrario, entre 1986 y 2015 se observa el incremento gradual y significativo en los caudales máximos anuales, aunque dicho incremento no responde necesariamente a una conexión lineal (Figura 3b). Se identifican, además, puntos de cambio en la media en 1984 y las décadas de 1970 y 1990, que coinciden en el primer momento con la puesta en operación del embalse; el resto, con cambios de fase de la PDO, una alta tasa de ocurrencia de eventos ENSO (cinco reportados en las décadas de 1970 y 1980) (Wolter \& Timlin, 1998), e inundaciones históricas del río Cauca en 1971, 1974, 1975, 1982, 1984, 1988, 1997, 1999, 2008, 2010 y 2011 (Enciso et al., 2016), que afectan la media de la serie temporal.

Tabla 1. Resultados de las pruebas de hipótesis de homogeneidad para los caudales diarios máximos anuales en el río Cauca.

\begin{tabular}{|c|c|c|c|c|c|c|c|c|}
\hline \multicolumn{3}{|c|}{ Estación } & \multicolumn{3}{|c|}{ La Balsa } & \multicolumn{3}{|c|}{ Juanchito } \\
\hline Serie & Prueba & Periodo & $65-15$ & RN & RA & $65-15$ & RN & RA \\
\hline \multirow{4}{*}{$Q_{\text {máx }}$} & \multirow[t]{2}{*}{$M-K$} & $\tau$ & $-0.33^{*}$ & -0.38 & -0.04 & -0.06 & -0.02 & $0.22^{*}$ \\
\hline & & Sig. & $\mathrm{P} 1$ & P5 & NS & NS & NS & P10 \\
\hline & \multirow[t]{2}{*}{ Pet } & Año & 1984 & 1975 & 1991 & 1984 & 1975 & 1994 \\
\hline & & Sig. & $\mathrm{P} 1$ & P5 & NS & NS & NS & P5 \\
\hline
\end{tabular}

RN: 1965-1984; RA: 1986-2015; M-K: prueba Mann-Kendall; $\tau$ es el estadístico de la prueba y su signo indica el sentido de la pendiente; * indica que las pruebas de MannKendall incluyen procesos para retirar la dependencia serial; Sig.: significancia estadística; NS: no significativo; P1: significativo al 1\%; P5: al 5\%; P10: al 10\%; Pet: prueba Pettitt; año: momento de cambio brusco en la media. 
Tecnología y

Ciencias $\cong$ Agua
2020, Instituto Mexicano de Tecnología del Agua

Open Access bajo la licencia CC BY-NC-SA 4.0

(https://creativecommons.org/licenses/by-nc-sa/4.0/)
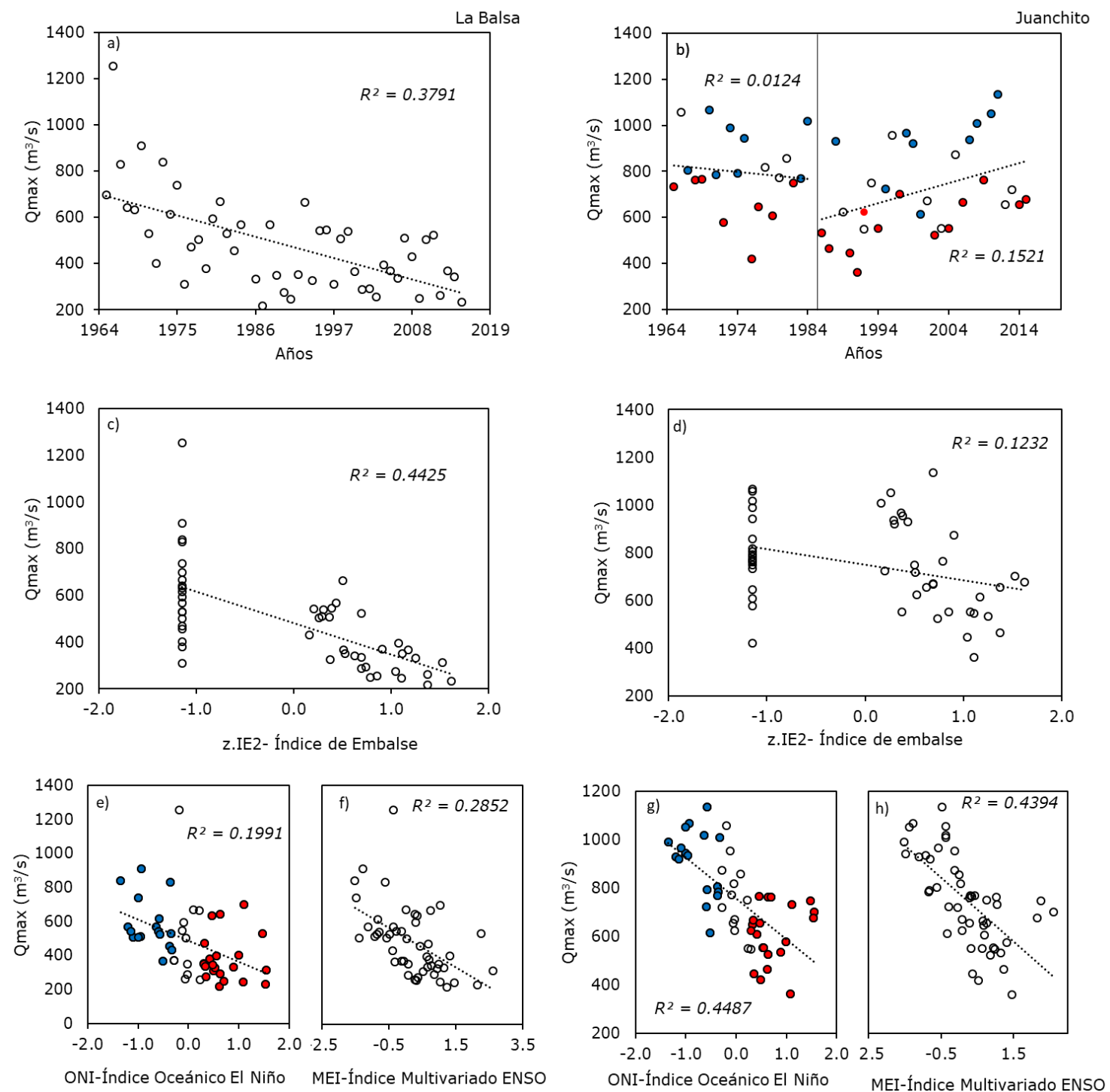

Figura 3. Variación de las crecidas anuales. De izquierda a derecha, La Balsa y Juanchito; a), b) evolución en el tiempo; c), d) conexión con el índice de embalse IE2; e) a h) relación con los índices ONI y MEI, resaltando en azul y rojo los caudales observados en años La Niña y El Niño, respectivamente. 
Ciencias $₫$ Agua
2020, Instituto Mexicano de Tecnología del Agua

Open Access bajo la licencia CC BY-NC-SA 4.0

(https://creativecommons.org/licenses/by-nc-sa/4.0/)

Los siguientes procesos físicos que pueden tener relación con las tendencias de aumento en el régimen de crecidas de la estación Juanchito entre 1986 y 2015 son:

- Los cambios en los usos del suelo en los últimos 30 años que afectan el escurrimiento de los ríos tributarios aguas abajo del embalse. Teniendo en cuenta que $43 \%$ del área de drenaje total a Juanchito corresponde a la cuenca del embalse, es coherente considerar que ríos tributarios importantes, que en la actualidad no son regulados, tienen un fuerte aporte al escurrimiento en la zona. También hay evidencias de aumento de la precipitación en dicha región. Ávila et al. (2019) analizaron las series temporales de índices extremos climáticos de precipitación en 39 estaciones meteorológicas del Valle Alto Cauca en el periodo 1970-2013. Identifican que para una significancia estadística $\alpha=0.10$ la precipitación acumulada en 1 y 5 días consecutivos aumenta entre 40 y $80 \mathrm{~mm}$ en el sur de la cordillera occidental de los Andes (entre el embalse y Juanchito). Esto coincide con las proyecciones de cambio climático que prevén, y un incremento de $6 \%$ de la precipitación total en los modelos climáticos del Valle del Cauca de los periodos 2011-2040, 2041-2070 y 2071-2100 (IDEAM-PNUD-MADS-DNP-Cancillería, 2015). Si además se tiene en cuenta que el fenómeno La Niña aumenta y prolonga los caudales altos, es de esperar que el efecto del embalse en el control de crecidas resulte limitado, y que las inundaciones se deban en buena medida al aporte de los ríos tributarios no regulados.

- Entre 1986 y 2015 se han registrado siete crecidas históricas durante años de La Niña (Enciso et al., 2016). De dichas crecidas, cuatro ocurren de forma simultánea con la fase fría de la Oscilación Decadal del Pacífico (PDO). De todas las crecidas recientes, las ocurridas en 2010-2011 se 
Tecnología y

Ciencias $\stackrel{\unlhd}{\unlhd}$ Agua
2020, Instituto Mexicano de Tecnología del Agua

Open Access bajo la licencia CC BY-NC-SA 4.0

(https://creativecommons.org/licenses/by-nc-sa/4.0/)

registran durante uno de los eventos La Niña más severos de la historia reciente (NOAA-ESRL-PSD, 2017).

- Hasta 1975, y luego entre 1999 y 2014 prevalecieron fases frías de la PDO (JMA \& WMO, 2017; Herzog, Martinez, Jorgensen, \& Tiessen, 2012; NOAA, 2017). Existen varias referencias sobre la acción simultánea de ENSO y la PDO en el régimen hidrológico en diversas regiones del mundo, que identifican que los efectos de los eventos El Niño/La Niña son más fuertes y ocupan una mayor área espacial global cuando ocurren en fase con periodos cálidos/fríos de la PDO (Garreaud, Vuille, Compagnucci, \& Marengo, 2009; Méndez, Ramírez, Cornejo, Zárate, \& Cavazos, 2011; Wang, Huang, He, \& Guan, 2015). Esto muestra la necesidad de más investigación sobre la acción conjunta de diferentes señales de variabilidad climática y la hidrología del suroccidente colombiano.

- Las modificaciones en las estaciones hidrométricas y/o en la forma de procesar los datos también pueden ser otra explicación a los cambios abruptos observados (Villarini et al., 2009a).

Los resultados obtenidos en la Tabla 1 resaltan la dificultad para aceptar/rechazar la hipótesis de i.i.d. del periodo 1965-2015 en la estación Juanchito, pues las pruebas no paramétricas aplicadas indican estacionariedad. Sin embargo, los cambios en la pendiente de las líneas de tendencia en los periodos RN y RA enmascaran el incremento continuo de las crecidas anuales en los últimos 30 años del periodo de análisis y, por lo tanto, la muestra RA amerita el análisis de frecuencias de crecidas no estacionario.

La Figura 3 muestra el comportamiento de las crecidas máximas anuales en función del tiempo; los índices de embalse y clima sugieren que aunque existe moderada correlación lineal $(\mathbf{0 . 3 0}<\boldsymbol{r}<\mathbf{0 . 7 0})$, la 02 
variabilidad explicada por los índices seleccionados es baja. Esto es comprensible, dada la compleja conexión entre los caudales y las variables climáticas, que no necesariamente se ajustan a modelos lineales; por lo tanto, puede ser necesario incluir funciones de suavizado en la modelación estadística no estacionaria.

\section{Modelos estadísticos no estacionarios del régimen de las crecidas en el río Cauca}

Los resultados de la modelación estadística de los caudales diarios máximos anuales de La Balsa (1965-2015) y Juanchito (1986-2015) se presentan a continuación.

La Tabla 2 muestra que el modelo estacionario (M0) en La Balsa se adhiere a la distribución Lognormal (LN2). La transformación Log ayuda a reducir la asimetría positiva de las observaciones. Luego, las crecidas en Juanchito se ajustan a la distribución Gamma; esta función tiene una forma suave y no requiere de la función Log para contrarrestar la asimetría; este último resultado es similar al obtenido por Enciso et al. (2016), quienes encuentran que los caudales diarios máximos anuales de Juanchito en el periodo 1986-2015 se ajustan de forma adecuada a una distribución Log Pearson III, que es una generalización de la distribución Gamma. Después se aprecia que el estadístico CIAG sugiere que los 
Tecnología y

Ciencias $₫$ Agua
2020, Instituto Mexicano de Tecnología del Agua

Open Access bajo la licencia CC BY-NC-SA 4.0

(https://creativecommons.org/licenses/by-nc-sa/4.0/)

modelos M1 presentan una menor pérdida de información. En cuanto a la calidad de ajuste, en todos los casos, los coeficientes de correlación de Filliben son mayores a los valores críticos, por lo tanto, se acepta la hipótesis de normalidad en los residuales y los modelos se ajustan de modo adecuado a las observaciones.

Tabla 2. Resumen de los modelos seleccionados y estadísticos de los residuales de GAMLSS de las crecidas máximas anuales en el río Cauca.

\begin{tabular}{|c|c|c|c|c|}
\hline $\begin{array}{c}\text { Variable } \\
\text { Estación } \\
\text { Periodo de } \\
\text { distribución }\end{array}$ & Modelo & Parámetros (error St.) & CIAG & CF \\
\hline \multirow{2}{*}{$\begin{array}{c}\text { Qmáx } \\
\text { La Balsa } \\
\text { 1965-2015 } \\
\text { LN2, } n=50\end{array}$} & M0 & $\mu_{1}=6.1[0.05], \sigma=-0.94[0.10]$ & 664.01 & 0.991 \\
\hline & M1 & $\begin{array}{l}\mu_{1}=6.10[0.04], \mu_{2}=-0.28 \operatorname{IE} 2[(0.04)] \\
\sigma=-1.30[0.10]\end{array}$ & $\underline{631.05}$ & $\underline{0.996}$ \\
\hline \multirow{2}{*}{$\begin{array}{c}\text { Qmáx } \\
\text { Juanchito } \\
\text { 1986-2015 } \\
\text { GA, } n=30\end{array}$} & MO & $\mu_{1}=6.58[0.05], \sigma=-1.31[0.13]$ & 405.85 & 0.988 \\
\hline & M1 & $\begin{array}{l}\mu_{1}=6.75[0.07], \mu_{2}=-0.12 p b(M E I)[0.05] \\
\mu_{3}=-0.21 I E 2[0.10], \sigma=-1.84[0.13]\end{array}$ & $\underline{385.68}$ & $\underline{0.993}$ \\
\hline
\end{tabular}

El modelo no estacionario de las distribuciones LN2 y GA tiene como función de enlace en el parámetro de localización $\ln \left(\mu_{t}\right)=\mu_{1}+\mu_{2} X_{i}+\cdots+\mu_{n} X_{m}$, en función de $X_{1}, X_{2}, \ldots X_{m}$ covariables; el parámetro de escala constante es expresado como $\ln \left(\sigma_{t}\right)=\sigma$. Además,

CIAG es el criterio de información de Akaike generalizado para un coeficiente de penalización $k=3$; CF es el coeficiente de correlación Filliben aplicado a los residuales

$$
\left(\text { Fill }_{n 50, \alpha 0.05}=0.977 ; \text { Fill }_{n 30, \alpha 0.05}=0.964\right) \text {. }
$$




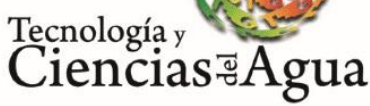

2020, Instituto Mexicano de Tecnología del Agua

Open Access bajo la licencia CC BY-NC-SA 4.0

(https://creativecommons.org/licenses/by-nc-sa/4.0/)

Debele, Bogdanowicz y Strupczewski (2017) sugieren que la selección de la función de distribución (fdp) es una de las decisiones más importantes para el adecuado análisis de los modelos. Consideramos que las pruebas de hipótesis establecen un ajuste adecuado, pero no necesariamente llevan a la selección de la mejor fdp. Para esto, los modelos GAMLSS aportan una amplia opción comparativa entre diferentes familias de fdp y al adherir la no estacionariedad. Sin embargo, es necesario el uso del criterio experto para seleccionaruna fdp que controle el efecto de la asimetría positiva característica de las series hidrológicas y un modelo sin sobreparametrización.

Sobre los modelos no estacionarios de mejor ajuste se encuentra:

a) La variabilidad temporal de las crecidas en la estación La Balsa es explicada a través del índice de embalse IE2. Esto se debe a que La Balsa posee la menor área de a portación total y al localizarse próxima a la salida del embalse predomina la regulación antrópica en los patrones de cambio de la serie.

b) Los caudales máximos en Juanchito 1986-2015 presentan una dependencia no lineal al índice MEI a través de suavizado por $p b()$ y lineal a IE2. Diferentes publicaciones indican que los GAMLSS con forzamientos climáticos resultan significativos para representar los cambios en la frecuencia y magnitud de las inundaciones en diferentes regiones del planeta (Machado et al., 2015; Obeyskera \& Salas, 2016; Villarini, Smith, Serinaldi, Ntelekos, \& Schwarz, 2012). En este trabajo las señales de ENSO y el índice de embalse de propuesto son adecuados para vincular los efectos antrópicos y climáticos a los patrones en las series de crecidas anuales del suroccidente de Colombia; además, es posible que en la 
medida que la estación se aleja del embalse, el efecto de la variabilidad climática resulte más significativo como variable explicativa.

Es importante resaltar que frente a las evidencias de no estacionariedad, es necesario adoptar metodologías que incorporen los patrones de cambio y permitan realizar un análisis comparativo de resultados. En este trabajo, los modelos no estacionarios en función de covariables (M1) muestran una mejor representación de la variabilidad de las series de tiempo, teniendo en cuenta que la mayoría de las observaciones se encuentra dentro de la banda de cuantiles de 1 a $99 \%$ de los modelos (Figura 4a y Figura 4b). Villarini, Serinaldi, Smith \& Krajewski (2009b) obtuvieron resultados similares al usar los GAMLSS para el análisis de las crecidas en una cuenca de EUA, mencionando que los modelos logran captar la amplia dispersión y no linealidad de los datos para percentiles entre el 5 y $95 \%$. 
Tecnología y

Ciencias $\approx$ Agua
2020, Instituto Mexicano de Tecnología del Agua

Open Access bajo la licencia CC BY-NC-SA 4.0

(https://creativecommons.org/licenses/by-nc-sa/4.0/) a)
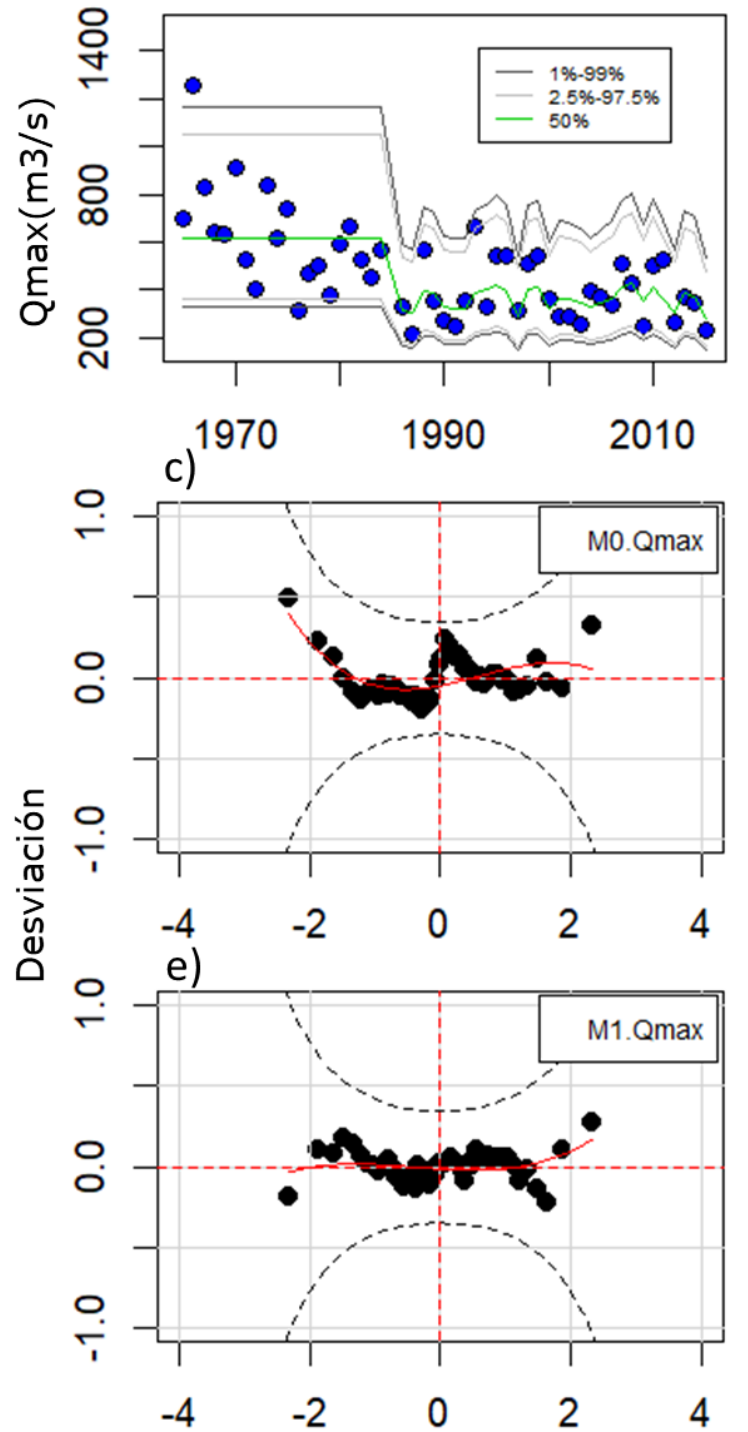

b)

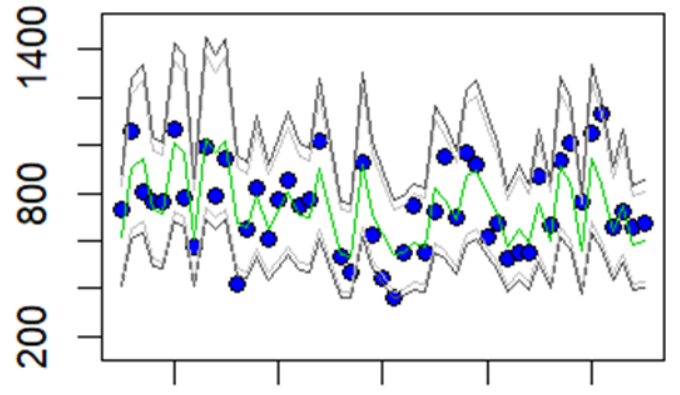

d)
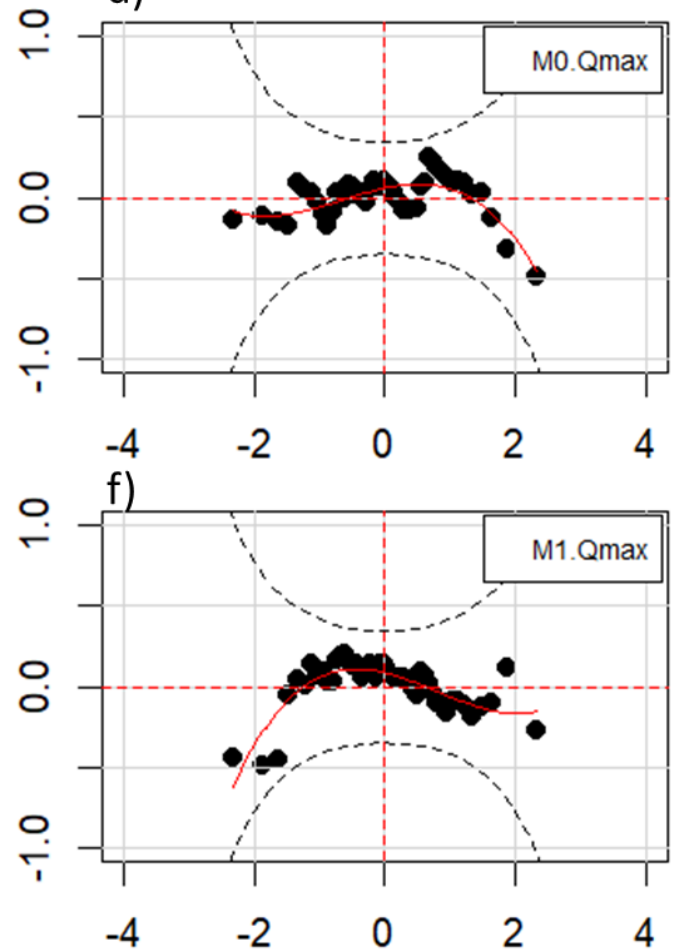

Cuantil normal unitario

Figura 4. Modelos estadísticos. De izquierda a derecha, las crecidas anuales en La Balsa y Juanchito; a) y b) la variación temporal de diferentes percentiles en el rango 1-99\% del modelo M1 (líneas continuas); los puntos azules corresponden a las observaciones.

Teóricamente se espera que entre 60 y $90 \%$ de las observaciones se ubique dentro del área cubierta por los percentiles 1 y $99 \%$ del

Tecnología y ciencias del agua, ISSN 2007-2422, 11(3), 27-77. DOI: 10.24850/j-tyca-2020-03- 
Tecnología y

Ciencias $₫$ Agua
2020, Instituto Mexicano de Tecnología del Agua

Open Access bajo la licencia CC BY-NC-SA 4.0

(https://creativecommons.org/licenses/by-nc-sa/4.0/)

modelo; c) a f) contienen los gráficos tipo gusano de los residuales de los modelos M0 y M1, respectivamente.

Los modelos no estacionarios M1 muestran para todos los percentiles graficados que en ciertos momentos la magnitud de las variables obtenidas es diferente a la estimada en condiciones estacionarias. Por ejemplo, en años La Niña se identifican incrementos en la magnitud de los caudales que pueden afectar los indicadores de riesgo de inundación, como el periodo de retorno y el riesgo de fallo para cierta vida útil. Sobre este resultado, López y Francés (2014), al evaluar las inundaciones en el noroeste de México, también encuentran una influencia significativa del fenómeno ENSO en la variabilidad interanual del régimen de crecidas, destacando aumentos de magnitud durante La Niña. A lo anterior se debe sumar la necesidad de reconocer que una limitación de los resultados de los modelos de covariable, es la incertidumbre asociada con el desconocimiento sobre el futuro, como el comportamiento de las variables explicativas más allá del periodo de registro (no existen proyecciones de largo plazo para los índices del ENSO que puedan incorporarse al poder predictivo de los modelos) y que otros procesos físicos (no considerados en el presente estudio) puedan resultar más significativos para describir la variabilidad de las crecidas.

La Figura 4 (c-f) evalúa la normalidad de los residuales de los diferentes modelos a partir de su configuración de un gráfico $Q-Q \sin$ tendencias, que suele tener forma de gusano; se busca que la línea continua roja (tendencia) se asemeje a una línea recta, paralela y cercana al eje horizontal. Por el contrario, cuando los residuales muestran configuraciones acentuadas en $\mathrm{S}$ o $\mathrm{U}$, indican alta asimetría y/o curtosis 
(Buuren \& Fredriks, 2001). En ambas estaciones es posible apreciar que los modelos cumplen la condición de normalidad. Sin embargo, de todos ellos, el modelo M1 de La Balsa presenta menos desviaciones a dicho supuesto.

\section{Análisis de cambios en el periodo de retorno}

La Figura 5 y la Tabla 3 muestran las variaciones obtenidas al estimar el periodo de retorno $T$ y riesgo de fallo $R$, para los modelos estacionarios y no estacionarios seleccionados. La Figura 5 ( de $T$ no estacionario en función de $T$ estacionario, siguiendo los métodos de tiempo de espera previsto (EWT) y del riesgo anual medio (AAR) (Salas et al., 2018). En este trabajo se establece que los caudales máximos anuales en La Balsa presentan una tendencia de disminución a lo largo del tiempo, de tal forma que las probabilidades de excedencia también sugieren un patrón decreciente. La Figura 4 (a) establece la variación de $T$ no estacionario, resaltando dos cosas. Primero, para $1 \leq T \leq 5$ años, el modelo no estacionario indica que $T_{E W T}<T$. Esto corresponde a un aumento en probabilidad de observar las crecidas menores. Sin embargo, al usar el método AAR no se aprecian diferencias entre $T$ no estacionario y $T \leq 20$ años, $\left(T_{A A R} \cong T\right)$. Dicha situación puede asociarse con que en los primeros 20 años de registros (1965-1984), la serie hidrológica no es afectada por el embalse, por lo que la media de la probabilidad de 
excedencia se mantiene constante y similar a la del modelo estacionario. Segundo, a partir de los puntos de inflexión mencionados, se aprecia $T_{E W T}>T$, y $T_{A A R}>T$. Así, la caída en la magnitud de las crecidas durante el régimen alterado (1986-2015) conlleva a la disminución en el riesgo hidrológico expresado como la probabilidad de observar una crecida igual a la de referencia o aumento en $T$ no estacionario. Dicho comportamiento de aumento y posterior disminución del riesgo hidrológico puede apreciarse de forma directa en la Figura 4 (c), donde el riesgo de fallo $R$ asociado con el caudal de crecida de $T=100$ años o probabilidad de observar, al menos una vez una crecida igual a la de referencia en un periodo $n$ años de vida útil. En el caso de La Balsa se observa $R_{E W T, A A R}$ mayor al estacionario para obras con $n<20$ años y un menor riesgo a partir de 30 años de vida útil. Otro aspecto a destacar es que los valores $R$ no estacionario en cada estación resultan idénticos sin importar el método de determinación, por lo que su uso podría ser una forma más directa de establecer el caudal de diseño de una obra hidráulica para un nivel de riesgo aceptable predefinido. 
Tecnología y

Ciencias $₫$ Agua
2020, Instituto Mexicano de Tecnología del Agua

Open Access bajola licencia CC BY-NC-SA 4.0

(https://creativecommons.org/licenses/by-nc-sa/4.0/)
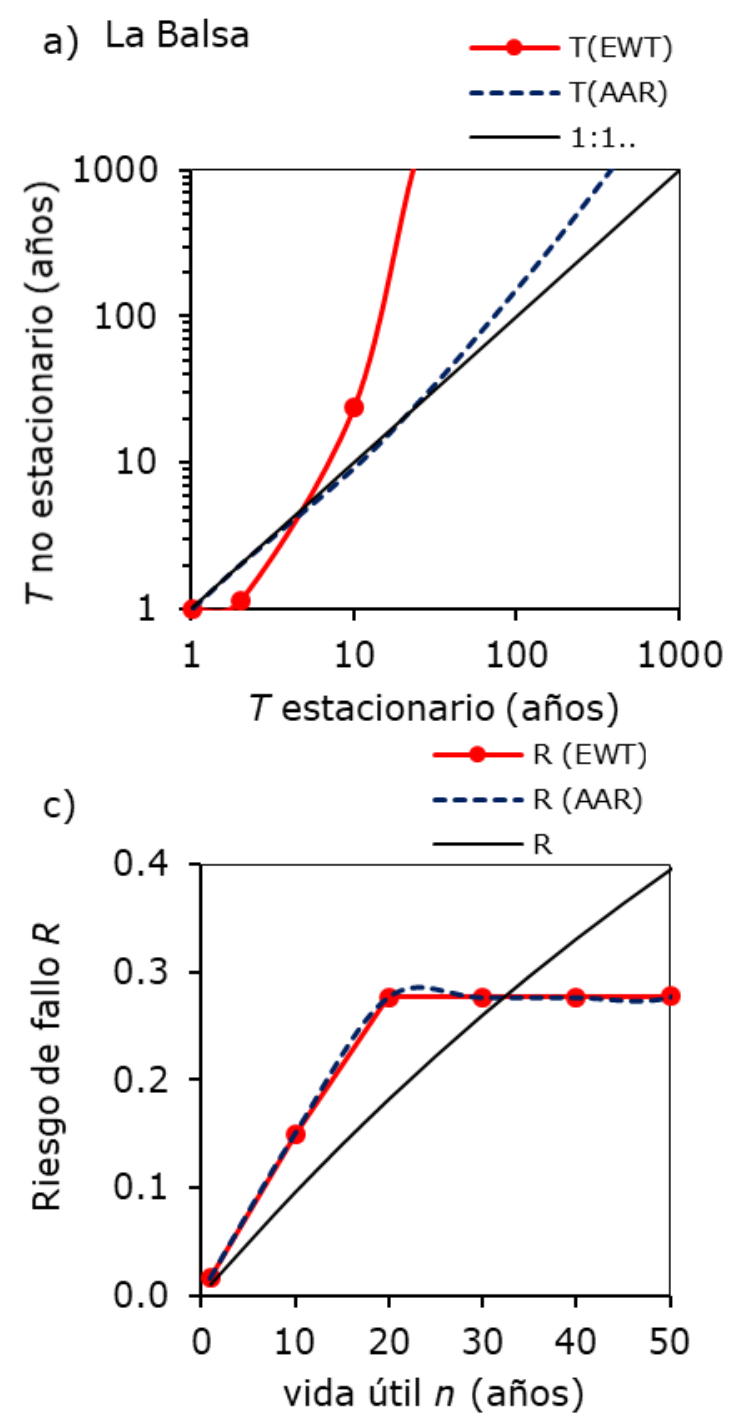

b) Juanchito

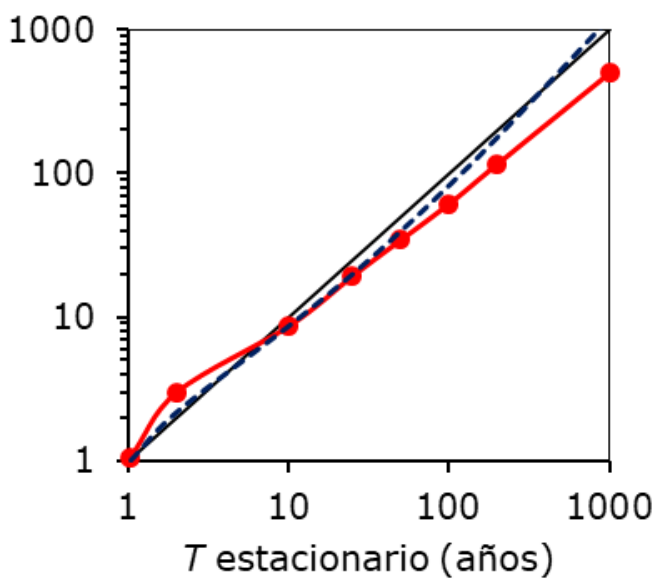

d)

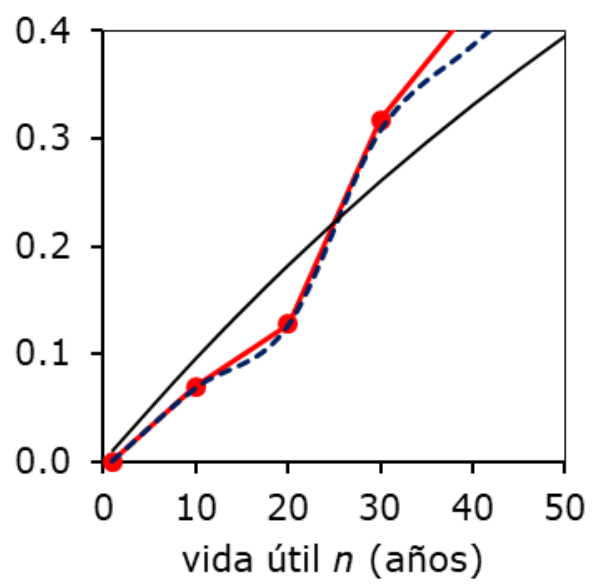

Figura 5. Variación del periodo de retorno y riesgo de fallo de los modelos estadísticos de La Balsa (izquierda) y Juanchito (derecha). Arriba, evolución de $T$ no estacionario en función de $T$ estacionario. Abajo, cambios del riesgo en función de la vida útil. 
Tecnología y

Ciencias $\stackrel{\varpi}{\triangleleft}$ gua
2020, Instituto Mexicano de Tecnología del Agua

Open Access bajo la licencia CC BY-NC-SA 4.0

(https://creativecommons.org/licenses/by-nc-sa/4.0/)

Tabla 3. Comparación entre el periodo de retorno estacionario y no estacionario para diferentes caudales de crecida.

\begin{tabular}{|c|c|c|c|c|c|c|c|c|}
\hline Estación & \multicolumn{8}{|c|}{ La Balsa } \\
\hline$Q_{\text {máx }}\left(\mathrm{m}^{3} / \mathrm{s}\right)$ & 212 & 445 & 735 & 883 & 995 & 1107 & 1221 & 1494 \\
\hline$T$ (años) & 1 & 2 & 10 & 25 & 50 & 100 & 200 & 1000 \\
\hline$T_{\text {(EWT) }}(a \tilde{n O S})$ & 1 & 1 & 24 & 1363 & 4410 & 7227 & 8829 & 9881 \\
\hline$T_{\text {(AAR)(años) }}$ & 1 & 2 & 9 & 26 & 62 & 155 & 402 & 4143 \\
\hline Estación & \multicolumn{8}{|c|}{ Juanchito } \\
\hline$Q_{\text {máx }}\left(\mathrm{m}^{3} / \mathrm{s}\right)$ & 397 & 700 & 975 & 1091 & 1171 & 1246 & 1316 & 1470 \\
\hline$T$ (años) & 1 & 2 & 10 & 25 & 50 & 100 & 200 & 1000 \\
\hline$T_{\text {(EWT)(años) }}$ & 1 & 3 & 9 & 19 & 34 & 61 & 116 & 497 \\
\hline$T_{\text {(AAR)(años) }}$ & 1 & 2 & 9 & 20 & 40 & 82 & 178 & 1194 \\
\hline
\end{tabular}

En el caso de las crecidas anuales en Juanchito entre 1986 y 2015 , se ha descrito una tendencia de aumento (Figura 3 (b)). Al comparar el periodo de retorno no estacionario, la Figura 5 (b) muestra: 1) cuando $1<$ $T<7$ años se obtiene $T_{E W T}>T$ y $T_{A A R}>T$. Por lo tanto, el modelo de covariables indica que las crecidas más frecuentes poseen menor probabilidad de ocurrencia, en comparación con el modelo estacionario. A partir de $T$ siete años ocurre lo contrario: $T_{E W T}<T$ y $T_{A A R}<T$. Aunque gráficamente dicha diferencia es modesta, la disminución de $T_{E W T}$ frente a $T$ oscila entre 14 y $50 \%$, mientras que entre $T_{A A R}$ y $T$ varía entre 15 y $39 \%$, con un leve incremento cuando $T$ es 1000 años. Dentro de las posibles explicaciones a la evolución de $T_{A A R}$ están la suave pendiente de cambio de los caudales máximos anuales, su significancia $(\alpha=0.10)$ y la corta longitud de la serie temporal (30 años). En todo caso, la información 
obtenida resalta un leve aumento en la probabilidad de ocurrencia de crecidas máximas extremas, lo que lleva a considerar que si bien la gestión del embalse tiene efectos positivos en las crecidas más comunes, existen factores externos (variabilidad climática, incremento en la precipitación, cambios en la cuenca no regulada, etc.) que inciden de manera negativa en el objetivo de regulación de las crecidas más raras y extremas. Similar información se presenta en la Figura 5 (d), en cuyo caso $R_{E W T, A A R}<T$ para una vida útil $n \leq 25$ años; pero a partir de dicho $n$, el riesgo de fallo del modelo no estacionario sobrepasa $R$ estacionario.

Todos los cambios en los indicadores de riesgo hidrológico ( $Q$ máx, $T$ y $R$ ) son de interés en la gestión de inundaciones a la altura de Juanchito, no sólo porque es la estación objetivo para el control de volúmenes en el embalse Salvajina sino porque el crecimiento urbanístico no planificado en la ciudad de Cali, como en muchas otras ciudades de países en vía de desarrollo, ha llevado a la intensa edificación en la llanura de inundación de ambas márgenes del río Cauca. Cerca de 900 mil habitantes de la ciudad se ubican en área de riesgo de inundación sobre la margen derecha del río Cauca. El control de crecidas es gestionado con la operación del embalse y diques laterales construidos para una crecida de $T=30$ años. A pesar de estas estructuras, los cambios identificados en el riesgo hidrológico se corrobora por caudales históricos observados en 2008, 2010, 2011 y 2017, así como por más de diez inundaciones en el área de Juanchito registradas en la última década (El País, 2011; Enciso et al., 2016), resaltando la necesidad de prever una gestión del riesgo de desastres naturales que incorpore una visión sistémica de los problemas e incluya los patrones de cambio asociados con la variabilidad climática. 
Tecnología y

Ciencias $\stackrel{\unlhd}{\unlhd}$ gua
2020, Instituto Mexicano de Tecnología del Agua

Open Access bajo la licencia CC BY-NC-SA 4.0

(https://creativecommons.org/licenses/by-nc-sa/4.0/)

\section{Conclusiones}

Los efectos de la operación del embalse y la variabilidad climática como patrones de alteración del régimen de crecidas anuales se analizan en este trabajo con el análisis de frecuencias de crecidas no estacionario, usando modelos GAMLSS. Las principales conclusiones son las siguientes:

Los registros de caudal diario entre 1965 y 2015 en la estación La Balsa señalan la disminución significativa $(\alpha=0.05)$, en la magnitud de las crecidas anuales. Los cambios bruscos y las tendencias graduales se relacionan con el cambio de fase en la PDO en la década de 1970, a fuertes sequías meteorológicas registradas en Colombia entre 1970 y 1990; pero sobre todo con la construcción y puesta en marcha del embalse en 1985.

Las crecidas máximas anuales en la estación Juanchito entre 1965 y 2015 presentan un patrón de incremento significativo $(\alpha=0.10)$ en los últimos 30 años de registros (1986-2015), que ha sido enmascarado por el comportamiento de las observaciones entre 1965 y 1984 . Esto puede explicar de manera parcial cómo, a pesar de la regulación de caudales de crecida, entre 1986 y 2015 se reportaron siete eventos de magnitud histórica, siendo el más severo el observado en 2010-2011, con un caudal de $1135 \mathrm{~m} / \mathrm{s}$. Lo anterior lleva a considerar no sólo la importancia de analizar tendencias en muestras de la serie hidrológica, sino además a 
reflexionar sobre la necesidad de ajustes en los planes de gestión de la cuenca.

El aumento en la magnitud de los caudales máximos anuales en Juanchito puede estar vinculado con la estrecha conexión entre las crecidas y eventos climáticos La Niña prolongados y fuertes, que en simultaneidad con la fase fría de la PDO fortalecen los núcleos de máxima precipitación en el área de estudio. Esta información muestra la necesidad de más investigación para establecerel tipo de conexión y los efectos que la acción conjunta de PDO y ENSO tienen sobre la hidrología del suroccidente colombiano. Además, es probable que los cambios en el uso del suelo en el área tributaria a Juanchito que no es regulada por el embalse ( $57 \%$ del área total) también afecten en las tendencias de incremento del escurrimiento.

Para todas las estaciones evaluadas, el estudio demuestra que el uso de términos aditivos mejora la descripción de los cambios en la frecuencia y magnitud de las inundaciones, aceptando la hipótesis de diferencias significativas entre los modelos estacionarios y no estacionarios.

La modelación estadística no estacionaria de los caudales diarios máximos anuales usa la covariable IE2 para describir los cambios en la estación La Balsa, y el conjunto de índices MEI e IE2 para explicar el comportamiento en el tiempo de las crecidas en Juanchito, logrando el objetivo del estudio. El nuevo índice de embalse propuesto contribuye a mejorar la representación de la variabilidad de las inundaciones. A pesar de la incertidumbre de los resultados, la nueva información puede 
contribuir a una selección más robusta de los caudales de diseño, y de los rangos de amenaza y riesgo admisibles.

Este artículo consigue valorar los métodos tiempo de espera previsto (EWT) y análisis de riesgo medio (AAR) para determinar el periodo de retorno y el riesgo de fallo no estacionarios; ambos indicadores logran captar de forma adecuada los cambios en la probabilidad de excedencia y por lo tanto funcionan como nuevos indicadores del riesgo hidrológico de inundaciones. Con independencia del método de determinación, se identifica para las crecidas menos frecuentes o más raras: a) un mayor $T$ y menor $R$ no estacionarios para las crecidas máximas en La Balsa; y b) en Juanchito, el aumento en la probabilidad de exceder un caudal máximo anual conlleva a la disminución de $T$ e incremento de $R$ no estacionarios.

Para finalizar, toda la información anterior es de interés en la gestión del riesgo de inundaciones en el Valle Alto del río Cauca. Por ejemplo, puede influir en el dimensionamiento de obras de protección frente a crecidas; en el diseño de obras de descarga del drenaje pluvial de Cali; conlleva a cambios en la zonificación del grado de amenaza y riesgo de inundación; puede tener implicaciones en el ordenamiento territorial; pero sobre todo, muestra la necesidad de incorporar tanto los efectos de ENSO como de la no estacionariedad en las reglas de operación del embalse Salvajina. No obstante, es importante mencionar que la gestión de las inundaciones debe tener un marco integral sobre las medidas disponibles, gestión de los recursos naturales, visiones de futuro y restricciones del entorno. 


\section{Agradecimientos}

A Colciencias-Becas Francisco José de Caldas; al Ministerio de Economía y Competitividad de España, proyecto de investigación TETISMED (CGL2014-58127-C3-3-R); a la Universidad del Valle, grupo de investigación IREHISA; a la Universitat Politècnica de València, España, y a la Universidad de Colima, México; al suministro de información de la Corporación Autónoma Regional del Valle del Cauca-CVC.

\section{Referencias}

Ahn, K., \& Palmer, R. (2016). Use of a nonstationary copula to predict future bivariate low flow frequency in the Connecticut River basin. Hydrological Processes, 30(19), 3518-3532. Recuperado de https://doi.org/10.1002/hyp.10876

Ávila, Á., Guerrero, F., Escobar, Y., \& Justino, F. (2019). Recent precipitation trends and floods in the Colombian Andes. Water, 11(2), 379. Recuperado de https://doi.org/10.3390/w11020379

Banco Mundial. (2014). Notas de política Colombia: hacia la paz sostenible, la erradicación de la pobreza y la prosperidad compartida. Washington, DC, EUA: Banco Mundial. Recuperado de http://www.bancomundial.org/content/dam/Worldbank/Feature Story/lac/Colombia Policy Notes pub SPA 11-7-14web.pdf

Buuren, S. V., \& Fredriks, M. (2001). Worm plot: A simple diagnostic device for modelling growth reference curves. Statistics in Medicine, 20(8), 1259-1277. Recuperado de https://doi.org/10.1002/sim.746 
Carvajal, Y., Jiménez, H., \& Materon, H. (1998). Incidencia del fenómeno ENSO en la hidroclimatología del valle del río Cauca-Colombia. Bull. I'Institut Français d'Études Andines, 27(3). Recuperado de http://www.redalyc.org/resumen.oa?id=12627336

Chow, V. T., Maidment, D. R., \& Mays, L. W. (1994). En: Suarez, M. (ed.). Hidrología aplicada, $2^{a}$ ed. Bogotá, Colombia: McGraw-Hill.

Córdoba, S., Palomino, R., Gámiz, S., Castro, Y., \& Esteban, M. (2015). Influence of tropical Pacific SST on seasonal precipitation in Colombia: Prediction using El Niño and El Niño Modoki. Climate Dynamics, 44(5-6), 1293-1310. Recuperado de https://doi.org/10.1007/s00382-014-2232-3

Debele, S. E., Bogdanowicz, E., \& Strupczewski, W. G. (2017). Around and about an application of the GAMLSS package to non-stationary flood frequency analysis. Acta Geophysica, 65(4), 885-892. Recuperado de https://doi.org/10.1007/s11600-017-0072-3

El País. (2011). Conozca el drama de los habitantes de Juanchito causado por las inundaciones. El País. Recuperado de https://www.elpais.com.co/cali/conozca-el-drama-de-los-habitantes-de-juanchitocausado-por-las-inundaciones.html

EM-DAT. (2018). The emergency events database - Universite Catholique de Louvain (UCL)-. Brussels, Belgium: CRED, D. Guha-Sapir. Recuperado de www.emdat.be

Enciso, A., Carvajal, Y., \& Sandoval, M. (2016). Hydrological analysis of historical floods in the upper valley of Cauca River. Ingeniería $y$ Competitividad, 57(1), 46-57. 
Escalante-Sandoval, C., \& Garcia-Espinoza, E. (2014). Analysis of annual flood peak records in Mexico. WIT Transactions on Information and Communication Technologies, 47, 49-60. Recuperado de https://doi.org/10.2495/RISK140051

García, M., Botero, A., Bernal, F., Ardila, E., \& Piñeros, A. (2012). Variabilidad climática, cambio climático y el recurso hídrico en Colombia. Revista de Ingeniería. Universidad de los Andes, 36(0121-4993), 60-64. Recuperado de http://ojsrevistaing.uniandes.edu.co/ojs/index.php/revista/article/ view/136

Garreaud, R., Vuille, M., Compagnucci, R., \& Marengo, J. (2009). Presentday South American climate. Palaeogeography, Palaeoclimatology, Palaeoecology, 281(3-4), 180-195. Recuperado de https://doi.org/10.1016/j.palaeo.2007.10.032

Gutiérrez, F., \& Dracup, J. (2001). The use of ENSO based streamflow forecasts for reservoir operations in Colombia. In: Bridging the gap (pp. 1-7). Reston, USA: American Society of Civil Engineers. Recuperado de https://doi.org/10.1061/40569(2001)164

Herzog, S. K., Martinez, R., Jorgensen, P., \& Tiessen, H. (eds.). (2012). Cambio climático y biodiversidad en los Andes tropicales. París, Francia: Instituto Interamericano para la Investigación del Cambio Global (IAI), São José dos Campos, y Comité Científico sobre los Problemas del Medio Ambiente (SCOPE).

IDEAM-PNUD-MADS-DNP-Cancillería, Instituto de Hidrología, Meteorología y Estudios Ambientales, Programa de las Naciones Unidas para el Desarrollo, Ministerio de Ambiente y Desarrollo 
Sostenible, Departamento Nacional de Planeación, \& Cancillería. (2015). Nuevos escenarios de cambio climático para Colombia 2011-2100: tercera comunicación nacional de cambio climático. Recuperado

de http://modelos.ideam.gov.co/media/dynamic/escenarios/documen to-nacional-departamental-2015.pdf

Jiménez-Cisneros, B. E., Oki, T., Arnell, N. W., Benito, G., Cogley, J. G., Döll, P., Jiang, T., \& Mwakalila, S. S. (2014). Freshwater resources. In: Field, C. B., Barros, V. R., Dokken, D. J., Mach, K. J., Mastrandrea, M. D., Bilir, T. E., Chatterjee, M., Ebi, K. L., Estrada, Y. O., Genova, R. C., Girma, B., Kissel, E. S., Levy, A. N., MacCracken, S., Mastrandrea, P. R., \& White, L. L. (eds.). Climate change 2014: Impacts, adaptation, and vulnerability. Part A: Global and sectoral aspects (pp. 229-269). Cambridge, UK, New York, USA: Cambridge University Press, Working Group II to the Fifth Assessment Report of the Intergovernmental Panel on Climate Change.

JMA \& WMO, Japan Meteorological Agency \& World Meteorological Organization. (2017). El Nino monitoring and outlook/TCC. Recuperado de http://ds.data.jma.go.jp/tcc/tcc/products/elnino/decadal/pdo.html

Khaliq, M. N., Ouarda, T. B. M. J., Ondo, J. C., Gachon, P., \& Bobée, B. (2006). Frequency analysis of a sequence of dependent and/or nonstationary hydro-meteorological observations: A review. Journal of Hydrology, 329(3-4), 534-552. Recuperado de https://doi.org/10.1016/j.jhydrol.2006.03.004 
Tecnología y

Ciencias $\stackrel{\unlhd}{\unlhd}$ Agua
2020, Instituto Mexicano de Tecnología del Agua

Open Access bajo la licencia CC BY-NC-SA 4.0

(https://creativecommons.org/licenses/by-nc-sa/4.0/)

Liang, Z., Jing, H. Y., Wang, Y., Binquan, L., \& Zhao, J. (2017). A sample reconstruction method based on a modified reservoir index for flood frequency analysis of non-stationary hydrological series. Stochastic Environmental Research and Risk Assessment, 32. Recuperado de https://doi.org/10.1007/s00477-017-1465-1

Lima, C., \& Lall, U. (2011). Spatio-temporal non-stationary flood frequency modeling: seasonal peak floods in southern Brazil modeled using pre- and concurrent Pacific and Atlantic Ocean conditions. AGU Fall Meeting Abstracts, 1, 06. Recuperado de http://adsabs.harvard.edu/abs/2011AGUFM.H24C.06L

López de la, J., \& Francés, F. (2013). Non-stationary flood frequency analysis in continental Spanish rivers, using climate and reservoir indices as external covariates. Hydrology and Earth System Sciences, 17(8), 3189-3203. Recuperado de https://doi.org/10.5194/hess-17-3189-2013

López de la, J., \& Francés, F. (2014). La variabilidad climática de baja frecuencia en la modelación no estacionaria de los regímenes de las crecidas en las regiones hidrológicas Sinaloa y Presidio San Pedro. Tecnología y ciencias del agua, 5(4), 79-101. Recuperado de http://www.redalyc.org/pdf/3535/353532522005.pdf

Machado, M., Botero, B., López de la, J., Francés, F., Díez, A., \& Benito, G. (2015). Flood frequency analysis of historical flood data under stationary and non-stationary modelling. Hydrology and Earth System Sciences, 19(6), 2561-2576. Recuperado de https://doi.org/10.5194/hess-19-2561-2015 
Mann, H. (1945). Non-parametric tests against trend. Econométrica, 13(3), 163-171. Recuperado de https://doi.org/10.2307/1907187

Matalas, N. C. (1997). Stochastic hydrology in the context of climate change. Climatic Change, 37, 89-101.

Méndez, J., Ramírez, A., Cornejo, E., Zárate, A., \& Cavazos, T. (2011). Teleconexiones de la oscilación decadal del Pacífico (PDO) a la precipitación y temperatura en México. Investigaciones Geográficas, (73). Recuperado de http://www.scielo.org.mx/scielo.php?script=sci_arttext\&pid=S018 8-46112010000300005

Milly, P. C. D., Betancourt, J., Falkenmark, M., Hirsch, R. M., Kundzewicz, Z. W., Lettenmaier, D. P., Stouffer, R. J., Dettinger, M. D., \& Krysanova, V. (2015). On critiques of "Stationarity is dead: Whither water management?" Water Resources Research, 51(9), 77857789. Recuperado de https://doi.org/10.1002/2015WR017408

Milly, P., Betancourt, J., Falkenmark, M., Hirsch, R., Kundzewicz, Z., Lettenmaier, D. , \& Stouffer, R. (2008). Stationarity is dead: Whither water management ? Science, 319(February), 573-574.

Montanari, A., \& Koutsoyiannis, D. (2014). Modeling and mitigating natural hazards: Stationarity is immortal! Water Resources Research, 50(12), 9748-9756. Recuperado de https://doi.org/10.1002/2014WR016092. Received

Moreno, J., Begueria, S., Garcés, L., \& García, J. (2003). Intensidad de las avenidas y aterramiento de embalses en el Pirineo Central español. Ería, (61), 159-167. Recuperado de https://doi.org/10.17811/ER.0.2003.159-167 
NOAA-ESRL-PSD, National Oceanic and Atmospheric Administration, Earth System Research Laboratory, \& Physical Sciences Division. (2017). Climate indices: monthly atmospheric and ocean time series. Recuperado de https://www.esrl.noaa.gov/psd/data/climateindices/list/

NOAA, National Oceanic and Atmospheric Administration. (2017). Pacific Decadal Oscillation (PDO)-Northwest Fisheries Science Center. Recuperado de https://www.nwfsc.noaa.gov/research/divisions/fe/estuarine/oeip/ ca-pdo.cfm

Obeyskera, J., \& Salas, J. D. (2016). Frequency of recurrent extremes under non-stationarity. Journal of Hydrologic Engineering, 21(5), 04016005.

Recuperado de https://doi.org/10.1061/(ASCE)HE.1943-5584.0001339

Pettitt, A. N. N. (1979). A non-parametric approach to the change-point problem. Applied Statistics, 28(2), 126-135. Recuperado de https://doi.org/10.2307/2346729

Poveda, G. (2004). La hidroclimatología de Colombia: Una síntesis desde la escala interdecadal. Revista de la Academia Colombiana de Ciencias Exactas, Físicas y Naturales, 28-107, 201-222. Recuperado de http://www.clas.ufl.edu/users/prwaylen/geo3280articles/Synthesis of Colombian hydrology.pdf

Poveda, G., \& Álvarez, D. (2012). El colapso de la hipótesis de estacionariedad por cambio y variabilidad climática: implicaciones para el diseño hidrológico en ingeniería. Revista de Ingeniería. Universidad de los Andes, 36(0121-4993), 65-76. 
Poveda, G., Jaramillo, L., \& Vallejo, L. (2014). Seasonal precipitation patterns along pathways of South American low-level jets and aerial rivers. Water Resources Research, 50(1), 98-118. Recuperado de https://doi.org/10.1002/2013WR014087

Poveda, G., Velez, J., Mesa, O., Hoyos, C., Mejía, F., Barco, O., \& Correa, P. (2002). Influencia de fenómenos macroclimáticos sobre el ciclo anual de la hidrología colombiana: cuantificación lineal, no lineal y percentiles probabilísticos. Meteorología Colombiana, (6), 121-130. Recuperado de http://www.geociencias.unal.edu.co/unciencias/datafile/user_23/file/METEOROLOGIA/13Poveda Clima Nuevo.pdf

Poveda, G., Waylen, P., \& Pulwarty, R. (2006). Annual and inter-annual variability of the present climate in northern South America and southern Mesoamerica. Palaeogeography, Palaeoclimatology, Palaeoecology, 234(1), 3-27. Recuperado de https://doi.org/10.1016/j.palaeo.2005.10.031

Puertas, O., \& Carvajal, Y. (2008). Incidencia de El Niño-Oscilación del Sur en la precipitación y la temperatura del aire en Colombia, utilizando el Climate Explorer. Revista Científica Ingeniería y Desarrollo, (23), 104-118. Recuperado de http://rcientificas.uninorte.edu.co/index.php/ingenieria/article/vie $\mathrm{w} / 2097 / 1346$

Rigby, R. A., \& Stasinopoulos, M. (2005). Generalized additive models for location, scale and shape. Journal of the Royal Statistical, 54, Part3(3), 507-554. Recuperado de https://doi.org/10.1111/j.14679876.2005.00510.x 
Rueda, O., \& Poveda, G. (2006). Variabilidad espacial y temporal del Chorro del Chocó y su efecto en la hidroclimatología de la región del Pacífico colombiano. Meteorología Colombiana, (501), 132-145.

Salas, J. D., Obeysekera, J., \& Vogel, R. M. (2018). Techniques for assessing water infrastructure for nonstationary extreme events: A review. Hydrological Sciences Journal, 63(3), 325-352. Recuperado de https://doi.org/10.1080/02626667.2018.1426858

Salas, J. D., \& Obeyskera, J. (2014). Revisiting the concepts of return period and risk for nonstationary hydrologic extreme events. Journal of Hydrologic Engineering, 19(3), 554-568. Recuperado de https://doi.org/10.1061/(ASCE)HE.1943-5584.0000820

Sandoval, M., \& Ramirez, C. (eds.). (2007). El río Cauca en su valle alto: un aporte al conocimiento de uno de los ríos más importantes de Colombia. Cali, Colombia: CVC, Corporación Autónoma Regional del Valle del Cauca. Recuperado de http://www.cvc.gov.co/index.php/servicio-al-ciudadano/inscribadepartamento-de-gestion-ambiental/77-recurso-hidrico/1386libro-rio-cauca

Sandoval, M., Ramirez, C., \& Santacruz, S. (2011). Optimización de la regla mensual de operación del embalse de Salvajina. Ingeniería de Recursos Naturales y del Ambiente, (6), 93-104. Recuperado de http://bibliotecadigital.univalle.edu.co/handle/10893/2596

Serinaldi, F. (2015). Dismissing return periods! Stochastic Environmental Research and Risk Assessment, 29(4), 1179-1189. Recuperado de https://doi.org/10.1007/s00477-014-0916-1 
Tecnología y

Ciencias $\stackrel{\unlhd}{\unlhd}$ Agua
2020, Instituto Mexicano de Tecnología del Agua

Open Access bajo la licencia CC BY-NC-SA 4.0

(https://creativecommons.org/licenses/by-nc-sa/4.0/)

Stasinopoulos, M., Rigby, R. A., Vlasios, V., Heler, G., \& Bastiani De, F. (2015). Flexible regression and smoothing the GAMLSS packages in $R$. Recuperado de http://www.gamlss.org/wpcontent/uploads/2015/07/FlexibleRegressionAndSmoothingDraft1.pdf

Vasiliades, L., Galiatsatou, P., \& Loukas, A. (2015). Nonstationary frequency analysis of annual maximum rainfall using climate covariates. Water Resources Management, 29(2), 339-358. Recuperado de https://doi.org/10.1007/s11269-014-0761-5

Villarini, G., Smith, J., Serinaldi, F., Ntelekos, A., \& Schwarz, U. (2012). Analyses of extreme flooding in Austria over the period 1951-2006. International Journal of Climatology, 32(8), 1178-1192. Recuperado de https://doi.org/10.1002/joc.2331

Villarini, G., \& Strong, A. (2014). Roles of climate and agricultural practices in discharge changes in an agricultural watershed in Iowa. Agriculture, Ecosystems \& Environment, 188, 204-211. Recuperado de https://doi.org/10.1016/j.agee.2014.02.036

Villarini, G, Smith, J. A., Serinaldi, F., Bales, J., Bates, P. D., \& Krajewski, W. F. (2009a). Flood frequency analysis for nonstationary annual peak records in an urban drainage basin. Advances in Water Resources, 32(8), 1255-1266. Recuperado de https://doi.org/10.1016/j.advwatres.2009.05.003

Villarini, G., Serinaldi, F., Smith, J. A., \& Krajewski, W. F. (2009b). On the stationarity of annual flood peaks in the continental United States during the 20th century. Water Resources Research, 45(8), 1-17. Recuperado de https://doi.org/10.1029/2008WR007645 
Wang, S., Huang, J., He, Y., \& Guan, Y. (2015). Combined effects of the Pacific decadal oscillation and El Niño-Southern Oscillation on global land dry-wet changes. Scientific Reports, 4(6651). Recuperado de https://doi.org/10.1038/srep06651

Wolter, K., \& Timlin, M. (1998). Measuring the strength of ENSO events: How does 1997/98 rank? Weather, 53(9), 315-324. Recuperado de https://doi.org/10.1002/j.1477-8696.1998.tb06408.x

Yue, S., \& Wang, C. Y. (2002). Applicability of prewhitening to eliminate the influence of serial correlation on the Mann-Kendall test. Water Resources Research, 38(6), 4-1. Recuperado de https://doi.org/10.1029/2001WR000861 